\title{
Electron vortex beams in nonuniform magnetic fields
}

\author{
Abhijeet Melkani $\odot^{*}$ and S. J. van Enk ${ }^{\dagger}$ \\ Department of Physics and Oregon Center for Optical, Molecular, and Quantum Science, University of Oregon, \\ Eugene, Oregon 97403-1274, USA
}

(Received 25 November 2020; accepted 28 June 2021; published 16 July 2021)

\begin{abstract}
We consider the quantum theory of paraxial nonrelativistic electron beams in nonuniform magnetic fields, such as the Glaser field. We find the wave function of an electron from such a beam and show that it is a joint eigenstate of two ( $z$-dependent) commuting gauge-independent operators. This generalized Laguerre-Gaussian vortex beam has a phase that is shown to consist of two parts, each being proportional to the eigenvalue of one of the two conserved operators and each having different symmetries. We also describe the dynamics of the angular momentum and cross-sectional area of any mode and how a varying magnetic field can split a mode into a superposition of modes. By a suitable change in the frame of reference, all of our analysis also applies to an electron in a quantum Hall system with a time-dependent magnetic field.
\end{abstract}

DOI: 10.1103/PhysRevResearch.3.033060

\section{INTRODUCTION}

The field of electron optics [1,2] was pioneered by Glaser $[3,4]$, based on similarities to light optics and the possibility of using electromagnetic fields as electron lenses. New life was injected into the field in the 21 st century [5-8] with the production and exploitation of electron vortex beams. Such beams, like their optical counterparts, are hollow and can carry a large amount of quantized angular momentum in the direction of their propagation.

In transmission electron microscopy vortex beams can be used to increase the resolution of the microscope to the atomic scale [9-12] and to probe chirality and magnetic dichroism in specimens [13-21]. The interaction of their orbital angular momentum degrees of freedom with external magnetic fields gives rise to interesting dynamics [22-25] which endows them with information-rich phase structures [26-29]. Electron vortex beams can also be used to study fundamental quantum-mechanical phenomena $[30,31]$ including imaging Landau states (previously hidden in condensed-matter systems) [32], performing Stern-Gerlach-like experiments [33-35], and achieving spin-filtering applications [36-38].

We focus here on the theoretical description of paraxial electron vortex beams, propagating along the $z$ direction in a nonuniform magnetic field pointing predominantly in the direction of propagation. The magnetic field is nonuniform in that its $z$ component is a function of $z$. In each transverse plane $z=$ const, the electron's spatial wave function can

\footnotetext{
*amelkani@uoregon.edu

†svanenk@uoregon.edu
}

Published by the American Physical Society under the terms of the Creative Commons Attribution 4.0 International license. Further distribution of this work must maintain attribution to the author(s) and the published article's title, journal citation, and DOI. be viewed as living in a Hilbert space of square-integrable functions on the two-dimensional $(x, y)$ plane and physical quantities are then represented as self-adjoint operators acting on that Hilbert space. Such an operator description based on the paraxial approximation is well known in optics $[39,40]$ and we show it is a convenient description for electron beams as well, yielding exact solutions of the paraxial equation.

In an inertial frame of reference traveling with the classical electron's velocity $v$ along the $z$ axis the electron is confined to a stationary plane and subject to a time-dependent magnetic field. Our solution thus also directly applies to a time-dependent quantum Hall system. We also ensure that the physical quantities we use to describe the Hall effect are gauge invariant and behave well in the limit of zero magnetic field.

In particular, this allows us to answer the following questions. Light waves whose electric field dependence on the azimuthal angle $\phi$ is given by $\exp (\operatorname{im} \phi)$ are known to possess orbital angular momentum $L_{z}$ in the $z$ direction equal to $m \hbar$ per photon. For a free electron the same is true, but for an electron in a nonzero magnetic field both $L_{z}$ and the local phase of its wave function are gauge dependent. So what does one measure when one forms an image of the electron's wave function? How is the measured phase related to the physical angular momentum of the electron and the form of the applied magnetic field?

This article is structured as follows. In Sec. II we derive the paraxial equation for a single electron in an arbitrary $z$-dependent magnetic field and give its exact solution. In Sec. III we show there are four physical quantities whose expectation values (as functions of $z$ ) satisfy a closed set of differential equations. We also construct two linear combinations of those four quantities that are conserved (as functions of $z$ ). Those two quantities are represented by two operators whose eigenvalue equations determine a complete basis for the electron's wave function for each $z$. In Sec. IV we provide explicit analytical solutions for three important cases, including the Glaser field which is a well-established model 
for a magnetic lens [41]. Those example solutions show what roles the two eigenvalues (i.e., the quantum numbers) of our two operators play in the solutions (one is that of determining phase factors and their symmetries). In Sec. V we show how an electron may make a transition from a single mode to a superposition of modes by propagating through a region with a varying magnetic field. In Sec. VI we point out connections of our theory to recent work on emulating gauge theories for charged particles by neutral atoms in laser fields, light beams in waveguides, etc. The theoretical descriptions of these systems are, by design, identical, but what one can measure in practice varies from one system to another.

\section{PARAXIAL EQUATION AND ITS SOLUTION}

Consider an electron of charge $-e$ and mass $m_{e}$ in a magnetic field $\boldsymbol{B}=B(z) \hat{z}-\frac{\rho}{2} \frac{d B(z)}{d z} \hat{\boldsymbol{\rho}} .{ }^{1}$ (The electron's spin degree of freedom is considered in Appendix A.) We use the symmetric gauge $\boldsymbol{A}=\frac{B(z)}{2} \rho \hat{\boldsymbol{\phi}}$, so the Hamiltonian is

$$
H=\frac{(\boldsymbol{p}+e \boldsymbol{A})^{2}}{2 m_{e}}=T_{\perp}-\frac{\hbar^{2}}{2 m_{e}} \frac{\partial^{2}}{\partial z^{2}} .
$$

Here $T_{\perp}$ is the transverse kinetic energy given in terms of the mechanical momentum $\boldsymbol{\pi}=\boldsymbol{p}+\boldsymbol{e A}$ by $T_{\perp}=\frac{\pi_{x}^{2}+\pi_{y}^{2}}{2 m_{e}}$. The electron beam is an approximate eigenfunction of the $p_{z}$ operator, so we look for solutions of the form

$$
\Psi(\rho, \phi, z)=e^{i k z} \chi(\rho, \phi, z),
$$

where $\chi(\rho, \phi, z)$ varies slowly with $z$ (i.e., $\left.\left|\frac{\partial^{2} \chi}{\partial z^{2}}\right| \ll k\left|\frac{\partial \chi}{\partial z}\right|\right)$. Hence we obtain

$$
\frac{\partial^{2} \Psi}{\partial z^{2}} \approx e^{i k z}\left(2 i k \frac{\partial \chi}{\partial z}-k^{2} \chi\right) .
$$

The time-independent Schrödinger equation $H \Psi=E \Psi$ can then, with the help of the paraxial approximation above, be written as

$$
i \hbar v \frac{\partial \chi}{\partial z}=\left(T_{\perp}-\Delta E\right) \chi=H_{\mathrm{eff}} \chi .
$$

Here $v=\frac{\hbar k}{m_{e}}$ is the velocity of the electron along the $z$ axis and $\Delta E=E-\frac{k^{2} \hbar^{2}}{2 m_{e}}$. We can get rid of the constant $\Delta E$ by the redefinition $\chi \rightarrow e^{i(\Delta E / \hbar v) z} \chi$. This is equivalent, within

\footnotetext{
${ }^{1}$ The extra radial term in the magnetic field is a consequence of the fact that $\boldsymbol{\nabla} \cdot \boldsymbol{B}$ needs to be zero.
}

the paraxial approximation, to setting $\Delta E$ to zero by adjusting $k$.

[Note that if we replace $z / v$ with time $t$ then the above equation is the time-dependent Schrödinger equation for an electron constrained to a plane and subject to the timevarying electromagnetic field $\boldsymbol{A}(t)$. This mapping to the time-dependent quantum Hall system and other systems will be discussed in Sec. VI.]

Now we define the Larmor frequency, which governs the precession of the electron in a magnetic field, as $\Omega(z)=\frac{e B(z)}{2 m_{e}}$. The transverse kinetic energy is then

$$
T_{\perp}=\frac{p_{x}^{2}+p_{y}^{2}}{2 m_{e}}+\frac{m_{e} \Omega(z)^{2} \rho^{2}}{2}+\Omega(z) L_{z},
$$

where $L_{z}$ is the $z$ component of the canonical angular momentum operator $L_{z}=(\boldsymbol{r} \times \boldsymbol{p})_{z}=-i \hbar \frac{\partial}{\partial \phi}$. This is not to be confused with the gauge-invariant mechanical angular momentum of the electron $(\boldsymbol{r} \times \boldsymbol{\pi})_{z}:=\mathcal{L}_{z}$ [42-44]. In the symmetric gauge the latter can be written as

$$
\mathcal{L}_{z}=L_{z}+m_{e} \Omega(z) \rho^{2} .
$$

One can also consider the $z$ component of the angular momentum of the electromagnetic field $\mathcal{L}_{z}^{\mathrm{em}}$, which for the present system turns out to be (see Table I) [46]

$$
\mathcal{L}_{z}^{\mathrm{em}}=-m_{e} \Omega(z) \rho^{2} .
$$

Equations (6) and (7) show that the $z$ component of the total angular momentum of the system $\mathcal{L}_{z}^{\text {tot }}=\mathcal{L}_{z}+\mathcal{L}_{z}^{\text {em }}$ has the same representation as $L_{z}$ in the chosen gauge. However, while $L_{z}$ is conserved only in the symmetric gauge, $\mathcal{L}_{z}^{\text {tot }}$, being a physical quantity, is conserved regardless of the chosen gauge. To find a second gauge-invariant quantity that is conserved as a function of $z$ we consider the operator equation

$$
\frac{d O}{d z}=\frac{\partial O}{\partial z}+\frac{i}{\hbar v}\left[T_{\perp}, O\right]=0 .
$$

In order to find a solution that is independent of the solution $L_{z}$ we first define the operator

$$
G_{\perp}=\frac{\boldsymbol{r}_{\perp} \cdot \boldsymbol{\pi}_{\perp}+\boldsymbol{\pi}_{\perp} \cdot \boldsymbol{r}_{\perp}}{2}=-i \hbar \rho \frac{\partial}{\partial \rho}-i \hbar,
$$

which is proportional to the rate of change of the electron's moment of inertia about the $z$ axis (see Table I). A gaugeinvariant operator that satisfies Eq. (8), and is thus conserved,

TABLE I. Gauge-invariant operators for a particle with mass $m_{e}$ and charge $q$. In the definition of $\mathcal{L}^{\text {em }}, \boldsymbol{A}^{\perp}$ refers to the transverse component of $\boldsymbol{A}$ which in Fourier space is orthogonal to $\boldsymbol{k}$ and which in position space satisfies $\boldsymbol{\nabla} \cdot \boldsymbol{A}^{\perp}=0$ [45]. In the symmetric gauge we have $\nabla \cdot \boldsymbol{A}=0$, so $\boldsymbol{A}=\boldsymbol{A}^{\perp}$.

\begin{tabular}{llcc}
\hline \hline$\hat{O}$ & Physical quantity described & Definition & Form under circular gauge, $\boldsymbol{A}=\frac{B(z)}{2} \rho \hat{\boldsymbol{\phi}}$ \\
\hline $\boldsymbol{\pi}$ & particle's linear momentum & $\boldsymbol{p}-q \boldsymbol{A}$ & $-i \hbar \frac{\partial}{\partial \rho} \hat{\boldsymbol{\rho}}-\left(\frac{q B(z)}{2} \rho+i \hbar \frac{1}{\rho} \frac{\partial}{\partial \phi}\right) \hat{\boldsymbol{\phi}}-i \hbar \frac{\partial}{\partial z} \hat{\boldsymbol{z}}$ \\
$\mathcal{L}$ & particle's angular momentum & $\boldsymbol{r} \times \boldsymbol{\pi}$ & $\boldsymbol{r} \times \boldsymbol{\pi}$ \\
$\mathcal{L}^{\mathrm{em}}$ & electromagnetic field's angular momentum & $q \boldsymbol{r} \times \boldsymbol{A}^{\perp}$ & $q \boldsymbol{\boldsymbol { r }} \times \boldsymbol{A}=\boldsymbol{r} \times \boldsymbol{p}-\boldsymbol{r} \times \boldsymbol{\pi}$ \\
$I_{z}$ & moment of inertia about the $z$ axis & $m_{e} \boldsymbol{r}_{\perp} \cdot \boldsymbol{r}_{\perp}$ & $m_{e} \rho^{2}$ \\
$G_{\perp}$ & $\frac{1}{2 m_{e}} \frac{d}{d t} I_{z}$ & $\frac{\boldsymbol{r}_{\perp} \cdot \boldsymbol{\pi}_{\perp}+\boldsymbol{\pi}_{\perp} \boldsymbol{r}_{\perp}}{2}=\boldsymbol{r}_{\perp} \cdot \boldsymbol{\pi}_{\perp}-i \hbar$ & $-i \hbar \rho \frac{\partial}{\partial \rho}-i \hbar$ \\
\hline \hline
\end{tabular}


is the Ermakov-Lewis invariant [47]

$$
\begin{aligned}
I(z) & =\frac{m_{e}}{2 \hbar}\left[w^{2}\left(T_{\perp}-\Omega \mathcal{L}_{z}\right)-v w \dot{w} G_{\perp}\right. \\
& \left.+\left(\Omega^{2} w^{2}+\frac{v^{2}}{2}\left(\dot{w}^{2}+w \ddot{w}\right)\right) m_{e} \rho^{2}\right],
\end{aligned}
$$

where $w(z)$ is any particular solution of the Ermakov-Pinney equation [48]

$$
\frac{4 \hbar^{2}}{m_{e}^{2} w^{4}(z)}-v^{2} \frac{\ddot{w}(z)}{w(z)}=\Omega^{2}(z),
$$

where the overdot signifies the derivative with respect to $z$. Note that this equation is invariant under three different transformations: $v \mapsto-v, \Omega \mapsto-\Omega$, and $z \mapsto-z$. We show later [see Eq. (18)] that $w(z)$ is proportional to the width of the beam. Hence, from now on we will refer to Eq. (11) as the lensing equation. While it is a nonlinear equation, its solutions can be written in terms of the solutions of the linear differential equation

$$
v^{2} \ddot{w}(z)+\Omega^{2}(z) w(z)=0,
$$

as shown in Ref. [49]. For $I(z)$ to be Hermitian we will only consider real-valued solutions of $w(z)$.

The fact that $\mathcal{L}_{z}^{\text {tot }}$ and $I(z)$ are conserved means that their eigenvalues are independent of $z$. Also, since they commute with the operator $\frac{\partial}{\partial z}+\frac{i}{\hbar v} T_{\perp}$ their eigenstates are the same (up to a phase factor) as the solutions $|\chi\rangle$ of Eq. (4). Therefore,

$$
\begin{gathered}
\mathcal{L}_{z}^{\text {tot }}|\chi(z)\rangle=\hbar l|\chi(z)\rangle, \\
I(z)|\chi(z)\rangle=\hbar(2 n+|l|+1)|\chi(z)\rangle,
\end{gathered}
$$

where $l$ is an integer and $n$ is a non-negative integer. We now have two new equations for the electron's wave function. Using an ansatz inspired by the form of a vortex beam in free space [50], we get as the solution [51]

$\chi_{n, l}=\frac{N}{w}\left(\frac{\rho}{w}\right)^{|l|} L_{n}^{|l|}\left(\frac{2 \rho^{2}}{w^{2}}\right) \exp \left(-\frac{\rho^{2}}{w^{2}}+i k \frac{\rho^{2} \dot{w}}{2 w}\right) e^{i l \phi-i \theta(z)}$.

Here $w(z)$ is a solution of Eq. (11), $L_{n}^{|l|}(x)$ is the associated Laguerre polynomial, and $N=\sqrt{\frac{2^{|l+1|}}{\pi} \frac{n !}{(n+|l|) !}}$ is the normalization constant. $\theta(z)$ has two terms, one proportional to the eigenvalue of $I(z)$ and the other to that of $\mathcal{L}_{z}^{\text {tot: }}$

$$
\theta(z)=(2 n+|l|+1) \frac{2 \hbar}{m_{e}} \int_{0}^{z} \frac{d z^{\prime}}{v} \frac{1}{w^{2}\left(z^{\prime}\right)}+l \int_{0}^{z} \frac{d z^{\prime}}{v} \Omega\left(z^{\prime}\right) .
$$

The second term is invariant with respect to the transformation $(l, \Omega) \rightarrow(-l,-\Omega)$ but under $(l, \Omega) \rightarrow(l,-\Omega)$ or $(l, \Omega) \rightarrow(-l, \Omega)$ it changes sign. The first term is invariant with respect to all three transformations. This difference in symmetry between modes with opposite vorticities $\pm l$ or opposite magnetic fields $\pm \Omega$ may be used to measure these two terms separately.

The phase of the electron's wave function is, however, gauge dependent. The experimentally measured image of the wave function is an interference pattern which depends only on a phase difference. The gauge-independent interference term is given by $\chi_{n, l}(x, y) \chi_{\text {ref }}^{*}(x, y)$, where $\chi_{\text {ref }}$ refers to a reference beam which either has traveled a different path or had different initial conditions or quantum numbers. If the two beams have different $l$ quantum numbers, the image (interference pattern) will rotate as the beams propagate forward [27].

The initial conditions on any mode (15) can be specified by the initial beam waist $\left.w(z)\right|_{z=0}$ and the initial radius of curvature of the wave front $\left.R(z)\right|_{z=0}=\left.\frac{w(z)}{\dot{w}(z)}\right|_{z=0}$ [52]. There is then only one unique solution of Eq. (11) once these initial conditions are specified.

\section{EXPECTATION VALUES OF PHYSICAL OBSERVABLES}

While the wave function (15) describes all there is to know about the problem, a simpler, and admittedly limited, description may also be useful. Such a description is provided by the expectation values of physical gauge-invariant observables.

This is not totally trivial as several of the usual physical observables employed to describe the quantum Hall effect (for example, the coordinates of the center of the cyclotron orbit) either diverge or vanish when $B \rightarrow 0$. All our observables have a finite nonzero limit when $B \rightarrow 0$ and therefore we can show explicitly how vortex beams that start off in free space can evolve to states that are known to describe the quantum Hall effect, i.e., Landau states.

The general solution of Eq. (4) can be written as a superposition of all the modes given by Eq. (15):

$$
\chi(\rho, \phi, z)=\sum_{n, l} c_{n, l} \chi_{n, l}(\rho, \phi, z) .
$$

If only one of the coefficients $c_{n, l}$ is nonzero, then the width of the beam as a function of $z$ is given by

$$
\left\langle\rho^{2}\right\rangle=2 \pi \int_{0}^{\infty} \rho d \rho\left|\chi_{n, l}\right|^{2} \rho^{2}=\left(n+\frac{|l|}{2}\right) w^{2}(z)
$$

and satisfies the lensing equation (11). Here we changed the variable of integration from $\rho$ to $\rho /|w(z)|$ and then used the properties of the Landau wave function $\chi_{n, l}^{(0)}$ [see Eq. (23)].

For the general case [Eq. (17)] we use Eq. (8) and take expectation values to get the following closed set of differential equations:

$$
\begin{aligned}
\frac{d}{d z}\left\langle T_{\perp}\right\rangle & =\dot{\Omega}(z)\left\langle\mathcal{L}_{z}\right\rangle, \\
\frac{d}{d z}\left\langle\rho^{2}\right\rangle & =\frac{2}{m_{e} v}\left\langle G_{\perp}\right\rangle, \\
\frac{d}{d z}\left\langle\mathcal{L}_{z}\right\rangle & =\dot{\Omega}(z) m_{e}\left\langle\rho^{2}\right\rangle+\frac{2 \Omega(z)}{v}\left\langle G_{\perp}\right\rangle, \\
\frac{d}{d z}\left\langle G_{\perp}\right\rangle & =\frac{2}{v}\left(\left\langle T_{\perp}\right\rangle-\Omega(z)\left\langle\mathcal{L}_{z}\right\rangle\right) .
\end{aligned}
$$

The closure of these differential equations can be traced to the fact that $G_{\perp}, \rho^{2}$, and $T_{\perp}$ are (gauge-invariant) generators of the Lie group $\mathrm{SU}(1,1)$. The system belongs to $\mathrm{U}(1) \times$ $\mathrm{SU}(1,1)$ with $\mathcal{L}_{z}^{\text {tot }}$, or equivalently $L_{z}$, generating $\mathrm{U}(1)$.

For an electron in a single mode, these four expectation values as functions of $z$ are fully determined by the two 
quantum numbers $n$ and $l$ and the values of $w(z)$ and its derivative, explicitly,

$$
\begin{aligned}
\left\langle T_{\perp}\right\rangle & =\hbar l \Omega+\frac{2 n+|l|}{2} m_{e}\left(\frac{v^{2}}{2}\left(\dot{w}^{2}+w \ddot{w}\right)+\Omega^{2} w^{2}\right), \\
\left\langle\rho^{2}\right\rangle & =\frac{2 n+|l|}{2} w^{2}, \\
\left\langle\mathcal{L}_{z}\right\rangle & =\hbar l+\frac{2 n+|l|}{2} m_{e} \Omega w^{2}, \\
\left\langle G_{\perp}\right\rangle & =\frac{2 n+|l|}{2} m_{e} v w \dot{w} .
\end{aligned}
$$

In terms of these we have

$$
\begin{aligned}
I(z) & =\frac{m_{e}}{\hbar(2 n+|l|)}\left(\left\langle\rho^{2}\right\rangle\left(T_{\perp}-\Omega \mathcal{L}_{z}\right)-\frac{\left\langle G_{\perp}\right\rangle}{m_{e}} G_{\perp}\right. \\
& \left.+\left(\left\langle T_{\perp}\right\rangle-\hbar l \Omega\right) \rho^{2}\right)
\end{aligned}
$$

for a single mode.

\section{EXAMPLE SOLUTIONS}

We have reduced the general problem to a linear differential equation (12) of one variable $w(z)$. While analytical solutions for many cases are known (see Refs. [53,54], for example), we discuss here only three cases of the most importance to electron optics.

\section{A. Constant magnetic field}

When the magnetic field is constant, $\Omega(z)=\Omega$, the general solution of the lensing equation (11) can be written as

$$
\begin{aligned}
w^{2}(z)= & w_{0}^{2}\left[\cos ^{2}\left(\frac{\Omega z}{v}\right)+\left(1+\frac{4 R_{0}^{2}}{w_{0}^{4} k^{2}}\right) \frac{v^{2}}{\Omega^{2} R_{0}^{2}} \sin ^{2}\left(\frac{\Omega z}{v}\right)\right. \\
& \left.+\frac{v}{\Omega R_{0}} \sin \left(\frac{2 \Omega z}{v}\right)\right],
\end{aligned}
$$

where $w_{0}=\left.w(z)\right|_{z=0}$ and $R_{0}=\left.R(z)\right|_{z=0}=\left.\frac{w(z)}{\dot{w}(z)}\right|_{z=0}$ determine the initial conditions. With the special choice of $w_{0}^{2}=$ $\frac{2 v}{k|\Omega|}=\frac{2 \hbar}{m_{e}|\Omega|}$ and $R_{0} \rightarrow \infty$ the solution $w(z)$ becomes constant. The corresponding wave functions (which were first derived by Landau [55]) are eigenstates of the Hamiltonian $T_{\perp}$,

$$
\chi_{n, l}^{(0)}=\frac{N}{w_{0}}\left(\frac{\rho}{w_{0}}\right)^{|l|} L_{n}^{|l|}\left(\frac{2 \rho^{2}}{w_{0}^{2}}\right) \exp \left(-\frac{\rho^{2}}{w_{0}^{2}}\right) e^{i l \phi-i \theta(z)} .
$$

The phase is $\theta(z)=(2 n+|l|+1) \frac{|\Omega| z}{v}+l \frac{\Omega z}{v}$.

\section{B. Free space}

The solution for free space is known to display surprisingly different behavior than that for a constant nonzero field [50,56]. This can also be seen in Eq. (22), which becomes undefined when $\Omega=0$. Taking the limit $\Omega \rightarrow 0$ gives

$$
w^{2}(z)=w_{0}^{2}\left[1+\left(\frac{1}{R_{0}^{2}}+\frac{4}{w_{0}^{4} k^{2}}\right) z^{2}+\frac{2 z}{R_{0}}\right] .
$$

It is now clear that it is impossible to have a choice of initial conditions for which the width $w(z)$ remains nonzero constant. Therefore, the radius of curvature $R(z)$ of the beam is finite and the beam will always diffract. The wave function of the electron is given by Eq. (15) where the phase can be written in terms of the Rayleigh diffraction length $z_{R}=\frac{k w_{0}^{2}}{2}$ as

$$
\begin{aligned}
\theta(z) & =(2 n+|l|+1)\left\{\tan ^{-1}\left[\frac{z}{z_{R}}\left(\frac{z_{R}}{R_{0}}+\frac{R_{0}}{z_{R}}\right)-\frac{z_{R}}{R_{0}}\right]\right. \\
& \left.+\tan ^{-1}\left(\frac{z_{R}}{R_{0}}\right)\right\} .
\end{aligned}
$$

\section{Glaser field}

The Glaser field

$$
B(z)=\frac{B_{0}}{1+(z-c)^{2} / a^{2}}
$$

is a very useful model for describing an electron lens. It is an approximation of the magnetic field near the axis of a single current-carrying coil [41]. The general solution of Eq. (11) for $\Omega(z)=\Omega_{0} /\left[1+(z-c)^{2} / a^{2}\right]$ is (see Appendix B for derivation)

$$
\begin{aligned}
w^{2}(z) & =w_{c}^{2} \frac{a^{2}+(z-c)^{2}}{a^{2}}\left[\cos ^{2}[\alpha(z)]+\frac{a}{\beta R_{c}} \sin [2 \alpha(z)]\right. \\
& \left.+\frac{a^{2}}{\beta^{2}}\left(\frac{1}{R_{c}^{2}}+\frac{4}{k^{2} w_{c}^{4}}\right) \sin ^{2}[\alpha(z)]\right],
\end{aligned}
$$

where

$$
\alpha(z)=\beta \arctan \left(\frac{z-c}{a}\right)
$$

and $\beta=\sqrt{1+a^{2} \Omega_{0}^{2} / v^{2}}$. Here the arbitrary initial conditions have been specified using both the width $w_{c}$ and the radius of curvature $R_{c}$ at $z=c$. The well-known focusing behavior of the Glaser field [41] is demonstrated in how the width $w(z)$ is minimized to $w_{c}$ at the center of the field $z=c$. This becomes clearer when, similar to the case of the constant magnetic field, we get rid of the oscillations by a special choice of $w_{c}=\frac{2 a}{\beta k}$ and $R_{c} \rightarrow \infty$, which yields

$$
w^{2}(z)=\frac{2}{k a} \frac{1}{\sqrt{1+a^{2} \Omega_{0}^{2} / v^{2}}}\left[(z-c)^{2}+a^{2}\right] .
$$

The phase of the beam for this special case is

$$
\begin{aligned}
\theta(z)= & \left((2 n+|l|+1) \sqrt{1+a^{2} \Omega_{0}^{2} / v^{2}}+\frac{l \Omega_{0} a}{v}\right) \\
& \times\left[\tan ^{-1}\left(\frac{z-c}{a}\right)+\tan ^{-1}\left(\frac{c}{a}\right)\right] .
\end{aligned}
$$

\section{MODE SPLITTING}

Analogous to the analysis of thin lenses in light optics, we are interested in the problem of the asymptotic final states obtained after the beam passes through an optical apparatus. We assume that the magnetic field is constant for the region 


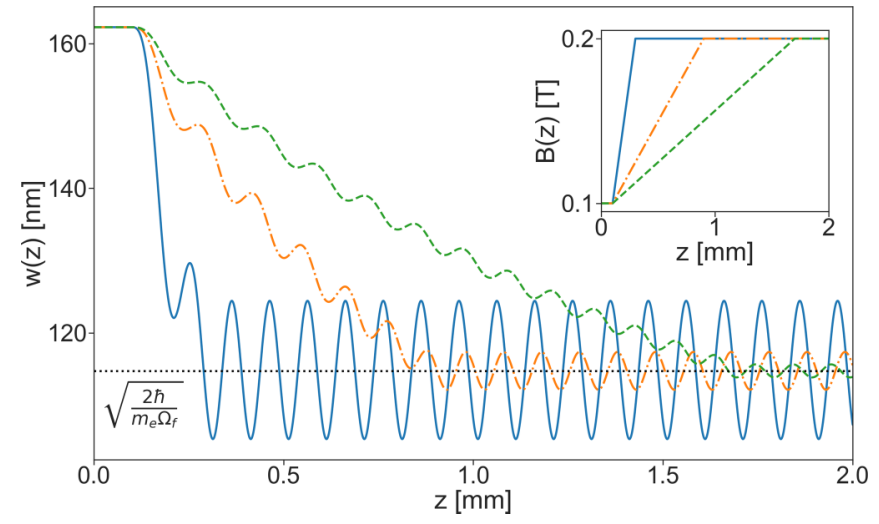

FIG. 1. Mode splitting by a ramp potential. A pure Landau mode (23) in a uniform magnetic field $B=0.1 \mathrm{~T}\left(\Omega_{i} \approx 8.8 \mathrm{GHz}\right)$ travels towards the positive- $z$ axis with speed $0.02 c$. Since it is a pure Landau mode $w(z)=\sqrt{\frac{2 \hbar}{m_{e} \Omega_{i}}}$ is constant. The beam is then subject to a ramp magnetic field (see the inset) in the region $z>0.1 \mathrm{~mm}$ until the magnetic field becomes $B=0.2 \mathrm{~T}\left(\Omega_{f} \approx 17.6 \mathrm{GHz}\right)$. The width of the beam $w(z)$ in the constant magnetic field region has now picked up an oscillating behavior and hence cannot be a pure Landau mode [which has a constant $w(z)=\sqrt{\frac{2 \hbar}{m_{e} \Omega_{f}}}$ shown by the black dotted line]. If the slope of the ramp is gradual (green dashed curve) we see an effective adiabatic behavior: The oscillations are relatively small. For the extreme case of slope approaching infinity the exact solution can be calculated using Eq. (31) with $g_{i}(z)=g_{f}(z)=1$.

$z<z_{i}$, varies in the region $z_{i}<z<z_{f}$, and then finally becomes constant again in the region $z>z_{f}$.

We take the initial wave function to have $w(z)=w_{i}=$ $\sqrt{\frac{2 \hbar}{m_{e}\left|\Omega_{i}\right|}}$ [and therefore $R(z)=\frac{w(z)}{\dot{w}(z)} \rightarrow \infty$ ]. This wave function corresponds to the one given in Eq. (23) and we will call such a wave function a pure Landau mode. After passing through the optical apparatus $\left(z_{i}<z<z_{f}\right)$, if the final wave function has $w(z)=\sqrt{\frac{2 \hbar}{m_{e}\left|\Omega_{f}\right|}}$ for $z>z_{f}$ then the Landau mode is unchanged and this is similar to adiabatic following.

However, if $w(z)$ takes the more general form [Eq. (22)], then the asymptotic wave function at $z \gg z_{f}$ will no longer be an eigenstate of the $z$ independent $H_{\text {eff }}\left(z_{f}\right)$ [even though it will still satisfy Eq. (4)]. This wave function can then be expressed as a superposition of Landau modes. In Fig. 1 we show such mode splitting for a ramp magnetic field (analytical solutions for which are known [53] in terms of Bessel functions).

While the general problem will require an exact solution of the lensing equation (11), here we follow Ref. [47] to provide an argument for why mode splitting can be expected. We first assume a convenient representation of the solution $w(z)$,

$$
w^{2}(z)= \begin{cases}\frac{2 \hbar}{m_{e}\left|\Omega_{i}\right|} g_{i}(z) & \text { if } z<0 \\ w_{z>z_{f}}^{2}(z) g_{f}(z) & \text { if } z>0\end{cases}
$$

where $w_{z>z_{f}}^{2}(z)$ is given by Eq. (22) with arbitrary constants $w_{0}$ and $R_{0}$ (these are no longer to be interpreted as the width and radius of the beam at $z=0$ ). Here $g_{i}$ and $g_{f}$ are continuous functions with continuous derivatives such that $g_{i}(z)=1$ for $z<z_{i}$ and $g_{f}(z)=1$ for $z>z_{f}$.
Since the wave function and its derivative need to be continuous, we demand that $w(z)$ and $\dot{w}(z)$ be continuous functions. However, $\Omega(z)$, and therefore $\ddot{w}(z)$, may be allowed to have a discontinuity at $z=0$. The Hamiltonian is therefore only piecewise constant.

Enforcing the continuity of $w(z)$ and $\dot{w}(z)$ at $z=0$ gives

$$
w_{0}^{2}=\frac{2 \hbar}{m_{e}\left|\Omega_{i}\right|} \frac{g_{i}(0)}{g_{f}(0)}, \quad R_{0}=\frac{2}{\frac{\dot{g}_{i}(0)}{g_{i}(0)}-\frac{\dot{g}_{f}(0)}{g_{f}(0)}} .
$$

The mode splits if

$$
w_{0}^{2}=\frac{2 \hbar}{m_{e}\left|\Omega_{i}\right|} \frac{g_{i}(0)}{g_{f}(0)} \neq \frac{2 \hbar}{m_{e}\left|\Omega_{f}\right|}
$$

or equivalently $\frac{g_{i}(0)}{\left|\Omega_{i}\right|} \neq \frac{g_{f}(0)}{\left|\Omega_{f}\right|}$. If one assumes that the magnetic field varies slowly such that $|v \dot{\Omega}(z)| \ll \Omega^{2}(z)$ [while staying within the paraxial regime of Eq. (3), $\left.\left|\frac{\partial^{2} \chi}{\partial z^{2}}\right| \ll \frac{m_{e} v}{\hbar}\left|\frac{\partial \chi}{\partial z}\right|\right]$, then the mode does not split [47].

\section{SUMMARY AND OUTLOOK}

In summary, we have derived the wave function of a paraxial electron beam moving in a nonuniform ( $z$-dependent) magnetic field. We found two operators, one angular momentum and the other denoted by $I(z)$ of Eq. (10), that are conserved as functions of $z$. Their eigenvalues are constant and their joint eigenstates form a complete basis for any transverse plane, which generalize the well-known LaguerreGaussian beams. We also constructed a set of four operators whose expectation values as functions of $z$ form a closed set of (differential) equations (19). These four quantities can then be expressed in terms of the two eigenvalues and the width $w(z)$ of the beam and its derivative $d w / d z$. Such a simple description is expected to have applications in lensing and directing beams. The phase of the beam is easily expressed as a function of $w(z)$, the magnetic field $B(z)$, and the two eigenvalues. This may be used for better image analysis of interference patterns in microscopy. Equation (11) for the width $w(z)$ can be solved analytically, and we provided complete solutions for the free field, a constant magnetic field, and the Glaser field, highlighting the relations between them. Finally, we also show how a Landau mode after passing through an optical apparatus can split into a superposition of Landau modes hinting at possible applications in production of modes with high angular momenta.

In the past few years many experimental applications have been achieved using electron vortex beams in magnetic fields. Their description could benefit from our theory especially in cases where previous analysis was done under assumptions of either adiabaticity $[25,34]$ or semiclassical dynamics $[5,33]$.

Finally, as previously noted, the system of an electron constrained to a plane and subject to a time-dependent potential $\boldsymbol{A}=\frac{B(t)}{2} \rho \hat{\boldsymbol{\phi}}$ leads to exactly the same equations (with $z / v$ replaced by $t$ ) as our Eqs. (4) and (5), namely,

$$
i \hbar \frac{\partial \chi}{\partial t}=\left(\frac{p_{x}^{2}+p_{y}^{2}}{2 m_{e}}+\frac{\mu \Omega(t)^{2} \rho^{2}}{2}+\Omega(t) L_{z}\right) \chi,
$$

with $\Omega(t)=\frac{e B(t)}{2 m_{e}}$. This corresponds to a time-varying magnetic field $\boldsymbol{B}(t)=\nabla \times \boldsymbol{A}=B(t) \hat{z}$ and an electric field $\boldsymbol{E}=$ 
$-\frac{\partial \boldsymbol{A}}{\partial t}=-\frac{B^{\prime}(t) \rho}{2} \hat{\boldsymbol{\phi}}$ due to Faraday's law. All of our analysis then also applies to this system of an electron in a quantum Hall system with time-dependent magnetic field after the mapping $z / v \rightarrow t$.

In fact, a similar equation can also be found in photonic topological insulators [see Eq. (2) of [57]]. Here the vector potential $\boldsymbol{A}$ does not represent an actual magnetic field but is an artificial gauge field that arises from being in a frame that rotates along with a helical waveguide.

Such artificial gauge fields are not just used in photonics to guide light beams [58], but may also be used to generate sonic Landau levels in metamaterials [59] (the gauge field being generated by nonuniform strains). For neutral atoms as well, it has been shown that laser fields can be used to mimic the dynamics of charged particles in a gauge field [60].

We expect our theoretical advances to be of relevance in each of these systems. Having different physical realizations of the same equation has the added benefit of being able to measure different quantities.

\section{ACKNOWLEDGMENTS}

We thank Ben McMorran, Will Parker, and Jayson Paulose for useful discussion.

\section{APPENDIX A: SPIN}

An actual physical electron also has spin which interacts with the magnetic field via $H_{B}=-\boldsymbol{\mu} \cdot \boldsymbol{B} \approx \frac{e}{m_{e}} \boldsymbol{S} \cdot \boldsymbol{B}$ (using $g \approx 2$ ). In our case we have

$$
H_{B} \approx 2 S_{z} \Omega(z)-\rho \dot{\Omega}(z) S_{\rho} .
$$

While the first term is comparable in magnitude to the spinless Hamiltonian, the second term is small since by the assumption of paraxial optics the beam is very close to the $z$ axis [61]

To incorporate spin one can construct two-component vector solutions from the scalar solutions we have provided [61].
The first term can be dealt with exactly and will appear as an independent spectator degree of freedom without any effect on the dynamics. The second term, which is small and couples spin to spatial degrees of freedom, can be dealt with perturbatively.

\section{APPENDIX B: SOLUTION OF THE LENSING EQUATION FOR THE GLASER FIELD}

To solve Eq. (11) for $\Omega(z)=\frac{\Omega_{0}}{1+(z-c)^{2} / a^{2}}$ we first perform the transformation $x=z-c$ and write the equation as

$$
\frac{d^{2} w(x)}{d x^{2}}+\frac{\Omega_{0}^{2} a^{4}}{v^{2}} \frac{w(x)}{\left(a^{2}+x^{2}\right)^{2}}-\frac{4}{k^{2}} \frac{1}{w^{3}(x)}=0 .
$$

As shown in Ref. [49], we can first solve the linear differential equation

$$
\frac{d^{2} w(x)}{d x^{2}}+\frac{\Omega_{0}^{2} a^{4}}{v^{2}} \frac{w(x)}{\left(a^{2}+x^{2}\right)^{2}}=0
$$

to get two independent solutions

and

$$
y_{1}(x)=\sqrt{a^{2}+x^{2}} \sin \left[\beta \arctan \left(\frac{x}{a}\right)\right]
$$

$$
y_{2}(x)=\sqrt{a^{2}+x^{2}} \cos \left[\beta \arctan \left(\frac{x}{a}\right)\right],
$$

where $\beta=\sqrt{1+a^{2} \Omega_{0}^{2} / v^{2}}$. Following the notation of Ref. [49], we define $u(x)=\frac{w_{c}}{a} y_{1}(x)+\frac{w_{c}}{\beta R_{c}} y_{2}(x)$, such that $u(0)=w_{c}$ and $\dot{u}(0)=\frac{w_{c}}{R}$, and $v(x)=y_{2}(x)$, so that $v(0)=$ 0 and $\dot{v}(0)=\beta$. The Wronskian $W$ of $u(x)$ and $v(x)$ is $u(x) \dot{v}(x)-\dot{u}(x) v(x)=w_{c} \beta$. Thus, using Eq. (2) of Ref. [49], the solution of the lensing equation for the Glaser field (B1) is

$$
w^{2}(x)=u^{2}(x)+\frac{4}{k^{2} W^{2}} v^{2}(x),
$$

with $w(x=0)=w_{c}$ and $\dot{w}(x=0)=\frac{w_{c}}{R_{c}}$. The above solution can be simplified further to finally get Eq. (27).
[1] P. Hawkes and E. Kasper, Principles of Electron Optics, 2nd ed. (Elsevier, Amsterdam, 2018).

[2] P. Grivet, Electron Optics, 2nd ed. (Elsevier, Amsterdam, 1972).

[3] W. Glaser, Strenge berechnung magnetischer linsen der feldform $H=H_{0} /(1+(z / a))^{2}$, Z. Phys. 117, 285 (1941).

[4] W. Glaser, Grundlagen der Elektronenoptik (Springer, Berlin, 2013).

[5] K. Y. Bliokh, Y. P. Bliokh, S. Savel'ev, and F. Nori, Semiclassical Dynamics of Electron Wave Packet States with Phase Vortices, Phys. Rev. Lett. 99, 190404 (2007).

[6] J. Verbeeck, H. Tian, and P. Schattschneider, Production and application of electron vortex beams, Nature (London) 467, 301 (2010).

[7] M. Uchida and A. Tonomura, Generation of electron beams carrying orbital angular momentum, Nature (London) 464, 737 (2010).

[8] B. J. McMorran, A. Agrawal, I. M. Anderson, A. A. Herzing, H. J. Lezec, J. J. McClelland, and J. Unguris, Electron vortex beams with high quanta of orbital angular momentum, Science 331, 192 (2011).
[9] F. Tamburini, G. Anzolin, G. Umbriaco, A. Bianchini, and C. Barbieri, Overcoming the Rayleigh Criterion Limit with Optical Vortices, Phys. Rev. Lett. 97, 163903 (2006).

[10] J. Verbeeck, P. Schattschneider, S. Lazar, M. Stöger-Pollach, S. Löffler, A. Steiger-Thirsfeld, and G. Van Tendeloo, Atomic scale electron vortices for nanoresearch, Appl. Phys. Lett. 99, 203109 (2011).

[11] J. Rusz, S. Muto, J. Spiegelberg, R. Adam, K. Tatsumi, D. E. Bürgler, P. M. Oppeneer, and C. M. Schneider, Magnetic measurements with atomic-plane resolution, Nat. Commun. 7, 12672 (2016).

[12] P. Schattschneider, B. Schaffer, I. Ennen, and J. Verbeeck, Mapping spin-polarized transitions with atomic resolution, Phys. Rev. B 85, 134422 (2012).

[13] S. Lloyd, M. Babiker, and J. Yuan, Quantized Orbital Angular Momentum Transfer and Magnetic Dichroism in the Interaction of Electron Vortices with Matter, Phys. Rev. Lett. 108, 074802 (2012).

[14] P. Schattschneider, S. Löffler, and J. Verbeeck, "Comment On Quantized Orbital Angular Momentum Transfer and Magnetic 
Dichroism in the Interaction of Electron Vortices with Matter," Phys. Rev. Lett. 110, 189501 (2013).

[15] J. Rusz, J.-C. Idrobo, and S. Bhowmick, Achieving Atomic Resolution Magnetic Dichroism by Controlling the Phase Symmetry of an Electron Probe, Phys. Rev. Lett. 113, 145501 (2014).

[16] J. Yuan, S. M. Lloyd, and M. Babiker, Chiral-specific electronvortex-beam spectroscopy, Phys. Rev. A 88, 031801(R) (2013).

[17] D. Pohl, S. Schneider, J. Rusz, and B. Rellinghaus, Electron vortex beams prepared by a spiral aperture with the goal to measure EMCD on ferromagnetic films via STEM, Ultramicroscopy 150, 16 (2015).

[18] P. Schattschneider, S. Löffler, M. Stöger-Pollach, and J. Verbeeck, Is magnetic chiral dichroism feasible with electron vortices? Ultramicroscopy 136, 81 (2014).

[19] T. Schachinger, S. Löffler, A. Steiger-Thirsfeld, M. StögerPollach, S. Schneider, D. Pohl, B. Rellinghaus, and P. Schattschneider, EMCD with an electron vortex filter: Limitations and possibilities, Ultramicroscopy 179, 15 (2017).

[20] A. Edström, A. Lubk, and J. Rusz, Elastic Scattering of Electron Vortex Beams in Magnetic Matter, Phys. Rev. Lett. 116, 127203 (2016).

[21] A. Edström, A. Lubk, and J. Rusz, Magnetic effects in the paraxial regime of elastic electron scattering, Phys. Rev. B 94, 174414 (2016).

[22] G. M. Gallatin and B. McMorran, Propagation of vortex electron wave functions in a magnetic field, Phys. Rev. A 86, 012701 (2012).

[23] C. R. Greenshields, R. L. Stamps, and S. Franke-Arnold, Vacuum Faraday effect for electrons, New J. Phys. 14, 103040 (2012).

[24] E. Karimi, L. Marrucci, V. Grillo, and E. Santamato, Spin-toOrbital Angular Momentum Conversion and Spin-Polarization Filtering in Electron Beams, Phys. Rev. Lett. 108, 044801 (2012).

[25] R. G. Littlejohn and S. Weigert, Adiabatic motion of a neutral spinning particle in an inhomogeneous magnetic field, Phys. Rev. A 48, 924 (1993).

[26] Y. Aharonov and A. Stern, Origin of the Geometric Forces Accompanying Berry's Geometric Potentials, Phys. Rev. Lett. 69, 3593 (1992).

[27] G. Guzzinati, P. Schattschneider, K. Y. Bliokh, F. Nori, and J. Verbeeck, Observation of the Larmor and Gouy Rotations with Electron Vortex Beams, Phys. Rev. Lett. 110, 093601 (2013).

[28] A. Lubk, G. Guzzinati, F. Börrnert, and J. Verbeeck, Transport of Intensity Phase Retrieval of Arbitrary Wave Fields Including Vortices, Phys. Rev. Lett. 111, 173902 (2013).

[29] L. J. Allen, H. M. L. Faulkner, M. P. Oxley, and D. Paganin, Phase retrieval and aberration correction in the presence of vortices in high-resolution transmission electron microscopy, Ultramicroscopy 88, 85 (2001).

[30] A. Béché, R. Van Boxem, G. Van Tendeloo, and J. Verbeeck, Magnetic monopole field exposed by electrons, Nat. Phys. 10, 26 (2014).

[31] I. P. Ivanov, D. Seipt, A. Surzhykov, and S. Fritzsche, Doubleslit experiment in momentum space, Europhys. Lett. 115, 41001 (2016).

[32] P. Schattschneider, T. Schachinger, M. Stöger-Pollach, S. Löffler, A. Steiger-Thirsfeld, K. Y. Bliokh, and F. Nori, Imaging the dynamics of free-electron Landau states, Nat. Commun. 5, 4586 (2014).

[33] H. Batelaan, T. J. Gay, and J. J. Schwendiman, Stern-Gerlach Effect for Electron Beams, Phys. Rev. Lett. 79, 4517 (1997).

[34] G. A. Gallup, H. Batelaan, and T. J. Gay, Quantum-Mechanical Analysis of a Longitudinal Stern-Gerlach Effect, Phys. Rev. Lett. 86, 4508 (2001).

[35] T. R. Harvey, V. Grillo, and B. J. McMorran, Stern-Gerlach-like approach to electron orbital angular momentum measurement, Phys. Rev. A 95, 021801(R) (2017).

[36] P. Schattschneider, V. Grillo, and D. Aubry, Spin polarisation with electron Bessel beams, Ultramicroscopy 176, 188 (2017).

[37] E. Karimi, V. Grillo, R. W. Boyd, and E. Santamato, Generation of a spin-polarized electron beam by multipole magnetic fields, Ultramicroscopy 138, 22 (2014).

[38] V. Grillo, L. Marrucci, E. Karimi, R. Zanella, and E. Santamato, Quantum simulation of a spin polarization device in an electron microscope, New J. Phys. 15, 093026 (2013).

[39] D. Stoler, Operator methods in physical optics, J. Opt. Soc. Am. 71, 334 (1981).

[40] S. J. van Enk and G. Nienhuis, Eigenfunction description of laser beams and orbital angular momentum of light, Opt. Commun. 94, 147 (1992).

[41] M. Szilágyi, Electron and Ion Optics (Springer, New York, 1998).

[42] Y. Kitadono, M. Wakamatsu, L. Zou, and P. Zhang, Role of guiding center in Landau level system and mechanical and pseudo orbital angular momenta, Int. J. Mod. Phys. A 35, 2050096 (2020).

[43] S. J. van Enk, Angular momentum in the fractional quantum Hall effect, Am. J. Phys. 88, 286 (2020).

[44] M. Wakamatsu, Y. Kitadono, L. Zou, and P. Zhang, The physics of helical electron beam in a uniform magnetic field as a testing ground of gauge principle, Phys. Lett. A 384, 126415 (2020).

[45] C. Cohen-Tannoudji, J. Dupont-Roc, G. Grynberg, and M. O. Scully, Photons \& Atoms: Introduction to Quantum Electrodynamics (Wiley-VCH, Weinheim, 1992).

[46] C. R. Greenshields, R. L. Stamps, S. Franke-Arnold, and S. M. Barnett, Is the Angular Momentum of an Electron Conserved in a Uniform Magnetic Field? Phys. Rev. Lett. 113, 240404 (2014).

[47] H. R. Lewis and W. B. Riesenfeld, An exact quantum theory of the time-dependent harmonic oscillator and of a charged particle in a time-dependent electromagnetic field, J. Math. Phys. 10, 1458 (1969).

[48] P. G. L. Leach and K. Andriopoulos, The Ermakov equation: A commentary, Appl. Anal. Discr. Math. 2, 146 (2008).

[49] E. Pinney, The nonlinear differential equation $y^{\prime \prime}+p(x) y+$ $c y^{-3}=0$, Proc. Am. Math. Soc. 1, 681 (1950).

[50] L. Allen, M. J. Padgett, and M. Babiker, in Progress in Optics, edited by E. Wolf (Elsevier, Amsterdam, 1999), Vol. 39, pp. 291-372.

[51] S. Menouar, M. Maamache, and J. R. Choi, The time-dependent coupled oscillator model for the motion of a charged particle in the presence of a time-varying magnetic field, Phys. Scr. 82, 065004 (2010).

[52] K. Y. Bliokh, P. Schattschneider, J. Verbeeck, and F. Nori, Electron Vortex Beams in a Magnetic Field: A New Twist on Landau Levels and Aharonov-Bohm States, Phys. Rev. X 2, 041011 (2012). 
[53] H. R. Lewis, Class of exact invariants for classical and quantum time-dependent harmonic oscillators, J. Math. Phys. 9, 1976 (1968).

[54] C. J. Eliezer and A. Gray, A note on the time-dependent harmonic oscillator, SIAM J. Appl. Math. 30, 463 (1976).

[55] L. Landau, Diamagnetismus der metalle, Z. Phys. 64, 629 (1930).

[56] K. Y. Bliokh, I. P. Ivanov, G. Guzzinati, L. Clark, R. Van Boxem, A. Béché, R. Juchtmans, M. A. Alonso, P. Schattschneider, F. Nori, and J. Verbeeck, Theory and applications of free-electron vortex states, Phys. Rep. 690, 1 (2017).

[57] M. C. Rechtsman, J. M. Zeuner, Y. Plotnik, Y. Lumer, D. Podolsky, F. Dreisow, S. Nolte, M. Segev, and A. Szameit,
Photonic Floquet topological insulators, Nature (London) 496, 196 (2013).

[58] Y. Lumer, M. A. Bandres, M. Heinrich, L. J. Maczewsky, H. Herzig-Sheinfux, A. Szameit, and M. Segev, Light guiding by artificial gauge fields, Nat. Photon. 13, 339 (2019).

[59] H. Abbaszadeh, A. Souslov, J. Paulose, H. Schomerus, and V. Vitelli, Sonic Landau Levels and Synthetic Gauge Fields in Mechanical Metamaterials, Phys. Rev. Lett. 119, 195502 (2017).

[60] J. Dalibard, F. Gerbier, G. Juzeliūnas, and P. Öhberg, Colloquium: Artificial gauge potentials for neutral atoms, Rev. Mod. Phys. 83, 1523 (2011).

[61] R. Jagannathan, Quantum theory of electron lenses based on the Dirac equation, Phys. Rev. A 42, 6674 (1990). 